University of Wollongong

Research Online

Faculty of Engineering and Information

Faculty of Engineering and Information

Sciences - Papers: Part A

Sciences

$1-1-2013$

\title{
Analytical solutions for filtration process based on constriction size concept
}

Vo Trong Nguyen

University of Wollongong, vtn838@uow.edu.au

Cholachat Rujikiatkamjorn

University of Wollongong, cholacha@uow.edu.au

Buddhima Indraratna

University of Wollongong, indra@uow.edu.au

Follow this and additional works at: https://ro.uow.edu.au/eispapers

Part of the Engineering Commons, and the Science and Technology Studies Commons

Research Online is the open access institutional repository for the University of Wollongong. For further information contact the UOW Library: research-pubs@uow.edu.au 


\title{
Analytical solutions for filtration process based on constriction size concept
}

\author{
Abstract \\ An analytical model is proposed to describe the filtration process applicable to a base soil-filter system. \\ The Navier-Stokes equations for porous media are used to capture the hydrodynamic behavior, whereas, \\ numerically, a new algorithm is proposed to solve the Navier-Stokes equation in a nonlinear form. The \\ various mixtures of base soil particles eroded and water flow within the system are computed using the \\ workenergy principle incorporating the constriction size of the filter. The model can assess the filtration \\ process through the flowrate and the accumulation and redistribution of fine particles within the filter. By \\ discretizing the base soil and filter domains into discrete elements, the model can predict the time- \\ dependent particle gradation of the filter for each element. Laboratory tests reported in other studies and \\ those conducted by the authors validate the model in relation to other available models.

\section{Keywords} \\ concept, size, constriction, process, solutions, filtration, analytical \\ Disciplines \\ Engineering | Science and Technology Studies

\section{Publication Details} \\ Nguyen, V., Rujikiatkamjorn, C. \& Indraratna, B. (2013). Analytical solutions for filtration process based on \\ constriction size concept. Journal of Geotechnical and Geoenvironmental Engineering, 139 (7), \\ 1049-1061.
}




\title{
Analytical Solutions for Filtration Process Based on Constriction Size Concept
}

\author{
Vo Trong Nguyen ${ }^{1}$; Cholachat Rujikiatkamjorn²; and Buddhima Indraratna, F.ASCE ${ }^{3}$
}

\begin{abstract}
An analytical model is proposed to describe the filtration process applicable to a base soil-filter system. The Navier-Stokes equations for porous media are used to capture the hydrodynamic behavior, whereas, numerically, a new algorithm is proposed to solve the Navier-Stokes equation in a nonlinear form. The various mixtures of base soil particles eroded and water flow within the system are computed using the workenergy principle incorporating the constriction size of the filter. The model can assess the filtration process through the flow rate and the accumulation and redistribution of fine particles within the filter. By discretizing the base soil and filter domains into discrete elements, the model can predict the time-dependent particle gradation of the filter for each element. Laboratory tests reported in other studies and those conducted by the authors validate the model in relation to other available models. DOI: 10.1061/(ASCE)GT.1943-5606.0000848. () 2013 American Society of Civil Engineers.
\end{abstract}

CE Database subject headings: Soil erosion; Filtration; Filters; Analytical techniques; Hydrodynamics.

Author keywords: Constriction size; Erosion; Filtration; Filter.

\section{Introduction}

Filters have been used in earth structures to prevent the erosion of base soils induced by seepage water. Since their first inception in the early 1920s by Terzaghi (Fannin 2008), filter and filtration problems have been investigated by numerous researchers using empirical and analytical methods. Examples of works using empirical methods are those of Vaughan and Soares (1982), Sherard et al. (1984a, b), Sherard and Dunnigan (1989), Lafleur et al. (1989), Honjo and Veneziano (1989), and Indraratna et al. (1996). Analytical models have been developed using probabilistic theories to determine the nature of the pore network or constriction sizes of filter media (Silveira et al. 1975; Kenney et al. 1985; Wittmann 1979; Witt 1993; Schuler 1996; Humes 1996; Indraratna et al. 2007; Raut and Indraratna 2008). These studies have made significant contributions toward the filter problems by suggesting particle size-based or constriction size-based criteria. However, because filtration is a time-dependent process where base soil particles are transported to and retained in the filter medium causing

${ }^{1}$ Ph.D. Candidate, Centre for Geomechamics and Railway Engineering, School of Civil, Mining and Environmental Engineering, Univ. of Wollongong, Wollongong City, NSW 2522, Australia.

${ }^{2}$ Senior Lecturer, Centre for Geomechamics and Railway Engineering, School of Civil, Mining and Environmental Engineering, Univ. of Wollongong, Wollongong City, NSW 2522, Australia; and Research Fellow, ARC Centre of Excellence in Geotechnical Science and Engineering, Univ. of Wollongong, Wollongong City, NSW 2522, Australia.

${ }^{3}$ Professor and Head, School of Civil, Mining and Environmental Engineering, Director, Centre for Geomechamics and Railway Engineering, Univ. of Wollongong, Wollongong City, NSW 2522, Australia; and Program Leader, ARC Centre of Excellence in Geotechnical Science and Engineering, Univ. of Wollongong, Wollongong City, NSW 2522, Australia (corresponding author). E-mail: indra@uow.edu.au

Note. This manuscript was submitted on March 17, 2012; approved on October 2, 2012; published online on November 17, 2012. Discussion period open until December 1, 2013; separate discussions must be submitted for individual papers. This paper is part of the Journal of Geotechnical and Geoenvironmental Engineering, Vol. 139, No. 7, July 1, 2013. CASCE, ISSN 1090-0241/2013/7-1049-1061/\$25.00. a gradual reduction in porosity, this mechanism needs to be studied in more detail.

Indraratna and Vafai (1997) and Locke et al. (2001) proposed analytical models to consider the filtration process by capturing particle transport within the porous media. These models can be used to evaluate the effectiveness of filters by assessing the accumulation of base soil and any change in the flow rate during filtration. However, there are some aspects of these models that need to be captured and extended. First, as analyzed by Silveira et al. (1975), Kenney et al. (1985), Witt (1993), Schuler (1996), Humes (1996), and Indraratna et al. (2007), the pore network or constriction sizes of filter media play an important role in the erosion of base soil particles. The larger the pore network, the more the base soils erode; hence, to consider the potential of base soil grains migrating into the filters, Indraratna and Vafai (1997) used the simple void network proposed by Kovacs (1981), which is suitable for uniform filters. Afterward, Locke et al. (2001) extended this model using the three-dimensional (3D) pore network suggested by Schuler (1996) that is considered to be more realistic and applicable to a broader range of filter gradations. Second, the hydrodynamic aspect applied to these models was simplified using Darcy's law that is restricted to laminar flow. Cedergren (1989) stated that the flow occurring within filter-size aggregate normally possesses a semiturbulent or a turbulent state. Hence, this study proposes an analytical model that captures particle transport within porous media to assess how effectively filters retain base soils. The model uses the Navier-Stokes equations to capture more realistic hydrodynamic conditions within the system where semiturbulent or turbulent flows may occur. It provides a physical explanation of how the new model better accounts for the forces applied to different sizes of particles by the relative motion of the fluid and the particles.

The algorithm suggested by Patankar (1980) has been revised in this study to include the nonlinear solution of the Navier-Stokes equations. This technique can provide a more realistic timedependent flow prediction in both steady-state and turbulent flow conditions. The accumulation and erosion of base soil particles within the filter media can be assessed using work-energy balance equations that incorporate the controlling constriction size concept proposed by Indraratna et al. (2007). The effect of energy dissipation is captured during the transport of slurry within the system. The 
outcomes of the model show that by simulating the flow rate, the accumulation of base soils, the infiltration depth within the filters, and the washout of base soil particles with effluent, the effectiveness of a filter with a given base soil can accurately be evaluated.

\section{Mathematical Description}

To investigate the effectiveness of a base soil-filter system, the concept of one-dimensional downward flow was adopted [Fig. 1(a)]. Downward water flow generates the velocity and pressure field that can exert hydraulic forces on base soil particles and transfer them into the filter medium. Based on the prescribed hydraulic conditions, base soil particles may be either retained within the filter at various depths or washed out of the system. To consider the principles of hydrodynamics within the system, the Navier-Stokes equation for porous media was used (Bouillard et al. 1989)

$$
\rho_{w} \frac{\partial(\in u)}{\partial t}+\rho_{w} u \frac{\partial(\in u)}{\partial x}=-\in \frac{\partial p}{\partial x}+\frac{\partial}{\partial x}\left[\left(\mu \frac{\partial(\in u)}{\partial x}\right)\right]+f_{b}
$$

where $\rho_{w}=$ water density; $u, p, \in$, and $\mu=$ velocity, pressure, porosity, and dynamic viscosity of water, respectively; and $f_{d}=$ body force per unit volume and can be defined as follows (Tsuji et al. 1993):

$$
f_{b}=-\beta u
$$

Generally, for Navier-Stokes equations, body forces account for any external forces acting on the fluid per unit volume (e.g., gravity). For flow within porous media, the body forces on the fluid are mainly the forces induced by the interaction between moving fluid and solid particles. In Eq. (2), $\beta$ can be calculated as follows (Ergun 1952):

$$
\beta=\frac{1-\epsilon}{d^{2} \epsilon^{2}}\left[150(1-\epsilon) \mu+1.75 \rho_{w} d u\right]
$$

where $d=$ diameter of the particles existing within the element. The mass balance equation can be expressed as

$$
\frac{\partial \in}{\partial t}+\frac{\partial(\in u)}{\partial x}=0
$$

Combining the Navier-Stokes equation [Eq. (1)] and mass balance equation [Eq. (4)], the term $[150(1-\epsilon) \mu]$ in Eq. (3) becomes dominant at lower Reynolds numbers (laminar state), and the second term $\left(1.75 \rho_{w} d u\right)$ becomes dominant at higher Reynolds numbers (turbulent state).

Water flow within the medium causes dislodgement and transport of base soil particles into the filter soil. Indraratna et al. (2007) pointed out that the constriction sizes of filters play an important role in controlling the erosion of base soils. Accordingly, particles of base soil that are smaller than the controlling constriction size $\left(D_{c}\right)$ are potentially erodible. The procedure for computing the controlling constriction size was described elsewhere by Indraratna et al. (2007). Once particles of base soil are eroded and transported into the filter medium, the mixture of base soil grains and water flow within the filter can be treated as homogenous slurry. To investigate any variation in the density of the slurry within the filter medium, the hydrodynamic work-energy principle was adopted. This principle states that the work done on a fluid system equals the change in the potential and kinetic energy of the system. Accordingly, the workenergy principle can be expressed as (Street et al. 1996)

$$
\frac{d W}{d t}=\frac{d E}{d t}
$$

where $W=$ work done and $E=$ energy.

To develop the work-energy equation, a control volume formed by two adjacent sections, numbered $i$ and $i+1$, that are perpendicular to the direction of flow within the filter medium was considered [Fig. 1(b)]. The slurry, entering the control volume at Section $i$, has density $\rho_{i}$ and average velocity $u_{i}$ and then leaving the control volume at Section $i+1$ has a density $\rho_{i+1}$ and a velocity $u_{i+1}$. The energy per unit mass in the control volume includes the potential energy ( $g z$, where $g$ is acceleration from gravity, $z$ is the height above the datum) and kinetic energy $\left(u^{2} / 2\right)$. Applying the Reynolds transport theorem (Street et al. 1996), the rate of change of energy of the system can be evaluated by

$$
\frac{d E}{d t}=\rho_{i+1}\left(g z_{i+1}+\frac{u_{i+1}^{2}}{2}\right) u_{i+1} \epsilon_{i+1}-\rho_{i}\left(g z_{i}+\frac{u_{i}^{2}}{2}\right) u_{i} \epsilon_{i}
$$

where $\rho, z, u$, and $\in=$ slurry density, height above the datum, velocity, and porosity, respectively. The subscripts in Fig. 1(b) denote the indexes for individual subsections.
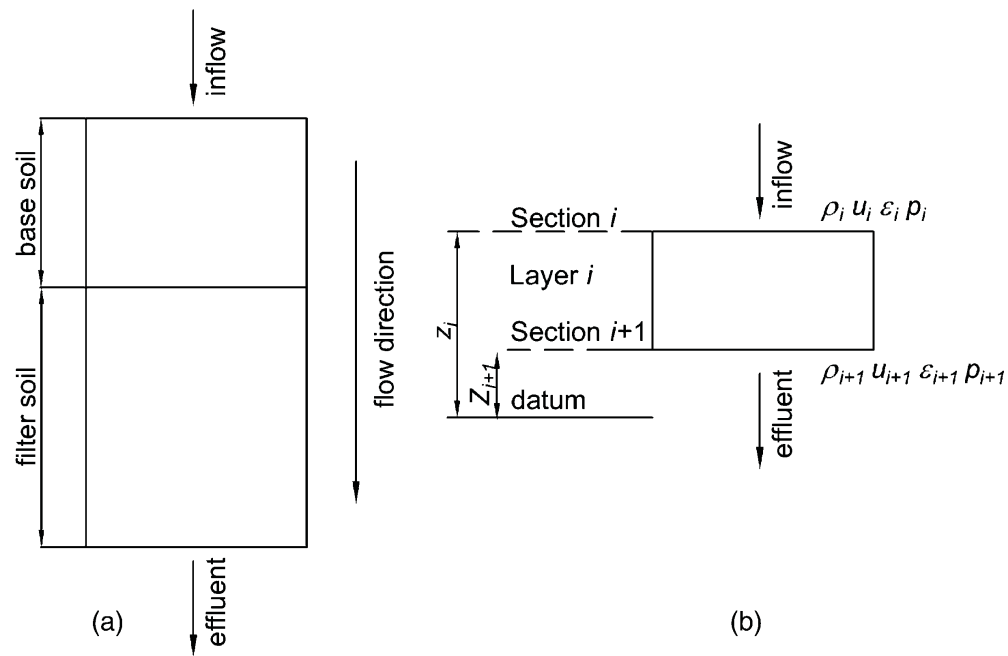

Fig. 1. (a) Typical base soil-filter system; (b) control volume for the consideration of work-energy equations 
In this case the work done on the fluid system can be divided into two forms: pressure work and shear work

$$
\frac{d W}{d t}=\frac{d W_{p}}{d t}+\frac{d W_{s}}{d t}
$$

where $W_{p}=$ pressure work caused by pressure forces via fluid entering and leaving the control volume; and $W_{s}=$ shear work done caused by shear forces acting on the system at control surface. The control surface is the total surfaces of all particles within the control volume. The rate of pressure work done by pressure forces can be expressed as

$$
\frac{d W_{p}}{d t}=p_{i} \epsilon_{i} u_{i}-p_{i+1} \epsilon_{i+1} u_{i+1}
$$

where $p_{i}$ and $p_{i+1}=$ pressures at Sections $i$ and $i+1$ in Fig. 1(b).

When flow is applied within the medium of particles, a stress field is exerted on the surfaces of the particles; hence, the work done within the control volume by stresses acting on the surfaces of all the particles can be determined as follows (Happel and Brenner 1965; Brenner 1958):

$$
\frac{d W_{s}}{d t}=-u_{L i} \sum_{m=1}^{n_{p}} F_{m}
$$

where $F_{m}=$ hydrodynamic force on the $m$ th particle within the layer; $n_{p}=$ number of particles within the layer; and $u_{L i}=$ velocity of water within the layer. Considering fluid flow in a porous medium, the energy loss occurs mainly because of the drag forces exerted on the particles. Besides, the drag forces are induced by the stress field on the particles surfaces (Holland and Bragg 1995). The work done by this mechanism can be simply calculated using Eq. $(9 a)$, where the hydrodynamic force $\left(F_{m}\right)$ is determined by (Holland and Bragg 1995)

$$
F_{m}=C_{d} S_{p} \rho u^{2} / 2
$$

where, $C_{d}=$ drag coefficient, $S_{p}=$ projected area, and $u=$ flow velocity.

However, because the slurry contains suspended particles, its viscosity $\left(\mu_{s}\right)$ is higher than clear water $(\mu)$. A relationship developed by Happel and Brenner (1965) can be used to determine the increase in viscosity caused by the interaction between the particles and pore walls and the slurry volumetric concentration $C_{v}$

$$
\frac{\mu_{s}}{\mu}=1+2.5 C_{v}\left[1+\frac{5 d D_{c}}{8\left(2 D_{c}-d\right)^{2}}\right]
$$

where $d=$ mean particle diameter of the slurry, and $D_{c}=$ controlling constriction size of the filter medium. The slurry volumetric concentration can be defined as the ratio between the volume of particles and the total slurry volume. At any time step, once Eq. (23) is solved, the slurry densities can be determined at the filter grid points. Accordingly, the slurry volumetric concentrations can be calculated using the known slurry densities.

This increase in viscosity causes an increased energy loss in the flow so that

$$
\frac{d W_{s}}{d t}=-\frac{\mu_{s}}{\mu} u_{L i} \sum_{m=1}^{n_{p}} F_{m}
$$

Combining Eqs. (5)-(11), the work-energy equation can be expressed as

$$
\begin{gathered}
\rho_{i+1}\left(g z_{i+1}+\frac{u_{i+1}^{2}}{2}\right) u_{i+1} \epsilon_{i+1}-\rho_{i}\left(g z_{i}+\frac{u_{i}^{2}}{2}\right) u_{i} \epsilon_{i} \\
=p_{i} \epsilon_{i} u_{i}-p_{i+1} \epsilon_{i+1} u_{i+1}-\frac{\mu_{s}}{\mu} u_{L i} \sum_{m=1}^{n_{p}} F_{m}
\end{gathered}
$$

At a given time step, the variation of slurry densities at the sections can be used to determine the accumulation or redistribution of base soil particles within each layer, In Fig. 1(b), for Layer $i$, the slurry densities at the incoming section (Section $i$ ) and outgoing section (Section $i+1)$ are $\rho_{i}$ and $\rho_{i+1}$, respectively. The incoming mass of slurry $\left(W_{i n}^{s}\right)$ for a unit area can be computed as follows:

$$
W_{\text {in }}^{s}=\rho_{i} u_{i} \in{ }_{i} \Delta t
$$

where $\Delta t=$ time interval.

The incoming mass of slurry includes the mass of particles and water

$$
W_{i n}^{s}=W_{i n}^{p}+W_{i n}^{w}
$$

where $W_{i n}^{p}$ and $W_{i n}^{w}=$ incoming masses of soil particles and water, respectively.

Eq. (14) then can be rearranged as follows:

$$
W_{i n}^{s}=W_{i n}^{p}+\rho_{w} V_{i n}^{w}=W_{i n}^{p}+\rho_{w}\left(V_{i n}^{s}-V_{i n}^{p}\right)
$$

Replacing volume terms with mass and density gives

$$
W_{i n}^{s}=W_{i n}^{p}+\rho_{w}\left(W_{i n}^{s} / \rho_{i}-W_{i n}^{p} / \rho_{p}\right)
$$

where $V_{i n}^{s}$ and $V_{i n}^{w}=$ incoming volumes of slurry and water, respectively; and $\rho_{i}, \rho_{p}$, and $\rho_{w}=$ densities of slurry at Section $i$, soil, and water, respectively. Hence, the mass of soil entering the layer can be determined as follows:

$$
W_{i n}^{p}=W_{i n}^{s} \frac{\rho_{p}}{\rho_{i}} \frac{\rho_{i}-1}{\rho_{i}-\rho_{w}}=u_{i} \in_{i} \Delta t \rho_{p} \frac{\rho_{i}-1}{\rho_{i}-\rho_{w}}
$$

Similarly, the outgoing mass of soil can be determined as follows:

$$
W_{\text {out }}^{s}=\rho_{i+1} u_{i+1} \in_{i+1} \Delta t
$$

or

$$
W_{\text {out }}^{p}=W_{\text {out }}^{s} \frac{\rho_{p}}{\rho_{i+1}} \frac{\rho_{i+1}-1}{\rho_{i+1}-\rho_{w}}=u_{i+1} \in_{i+1} \Delta t \rho_{p} \frac{\rho_{i+1}-1}{\rho_{i+1}-\rho_{w}}
$$

where $W_{\text {out }}^{s}$ and $W_{\text {out }}^{p}=$ outgoing mass of slurry and soil, respectively; and $\rho_{i+1}, \rho_{p}$, and $\rho_{w}$ are densities of slurry at Section $i+1$, soil, and water, respectively. Subsequently, the total mass $W^{p}$ of soil particles remaining in this layer can be calculated by

$$
W^{p}=W_{\text {in }}^{p}-W_{\text {out }}^{p}
$$

The mass of soil corresponding to a specific diameter $\left(d_{j}\right)$ accumulated within this layer can be computed based on the assumption that all eroded particles whose sizes are smaller than the controlling constriction size have an equal chance of being eroded or accumulated in the filter and thus

$$
W_{j}^{p}=P_{j} W^{p}
$$

where $P_{j}=$ percentage by mass of particle having diameter $d_{j}$ within the slurry. 


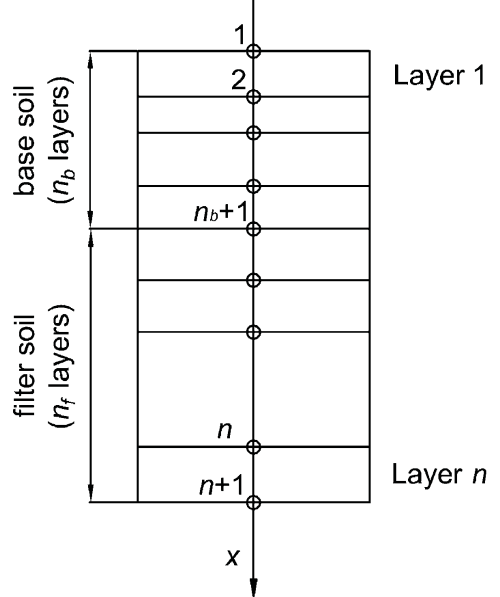

(a)

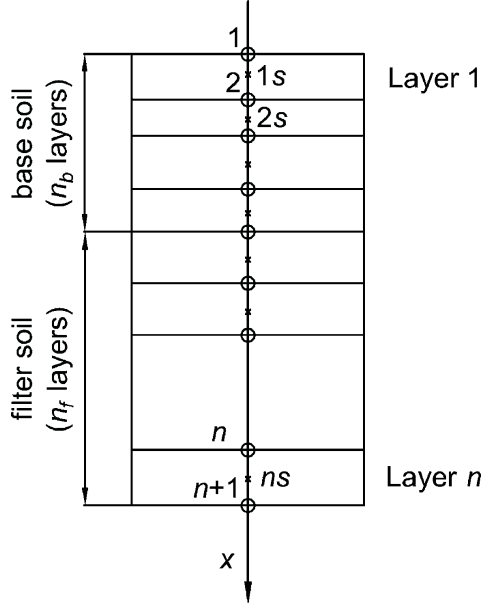

(b)

Fig. 2. (a) Discretization of the medium; (b) staggered grid (numbered $1 s$ to $n s$, where $s$ denotes staggered)

\section{Numerical Procedures}

The material domain was discretized by a grid of layers to study time-dependent filtration behavior [Fig. 2(a)]. Accordingly, the base soil-filter system was divided into $n$ layers that comprise a number of base soil layers $\left(n_{b}\right)$ and a number of filter soil layers $\left(n_{f}\right) . l_{i}$ is the corresponding length of Layer $i$. The indexes for points are also demonstrated in Fig. 2(a).

A numerical method (control-volume formulation taken from Patankar 1980) was applied to obtain discretization equations. Three consecutive points $(i, j$, and $k)$ of the system were considered for a given control volume. Normally, the faces of the control volume adjacent to Point $j$ are expressed in Fig. 3(a). However, to calculate the momentum equation of the flow field, this control volume appeared to be unstable; therefore, a staggered grid was used, as shown in Fig. 2(b) (Patankar 1980; Tannehill et al. 1997). Accordingly, a staggered point $(j s)$ is located in the middle of Point $j$ and the next point (i.e., Point $k$ ). The velocity for the staggered locations can be calculated. The discretization equation is now derived by integrating Eq. (1) over the control volume shown in Fig. 3(b) and over the time interval from $t$ to $t+\Delta t$. Thus

$$
\begin{aligned}
& \int_{j}^{k} \int_{t}^{t+\Delta t} \rho_{w} \frac{\partial(\in u)}{\partial t} d t d x+\int_{t}^{t+\Delta t} \int_{j}^{k} \rho_{w} u \frac{\partial(\in u)}{\partial x} d x d t \\
& =-\int_{t}^{t+\Delta t} \int_{j}^{k} \in \frac{\partial p}{\partial x} d x d t+\int_{t}^{t+\Delta t} \int_{j}^{k} \frac{\partial}{\partial x}\left[\mu \frac{\partial(\in u)}{\partial x}\right] d x d t \\
& +\int_{t}^{t+\Delta t} \int_{j}^{k} f_{b} d x d t
\end{aligned}
$$

In this expression, a fully implicit scheme and piecewise-linear profile were used to obtain the discretization equations, and a nonlinear form via a semiimplicit method for pressure-linked equation (SIMPLE) was used in this study to solve the Navier-Stokes equations (Patankar 1980). The revised algorithm for the nonlinear solution is presented in the Appendix. To demonstrate the advantage of using the nonlinear form, a comparison of the linear and nonlinear

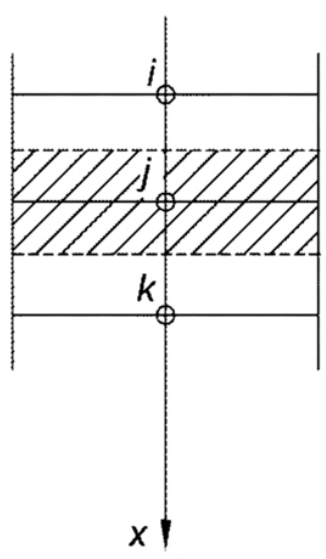

(a)

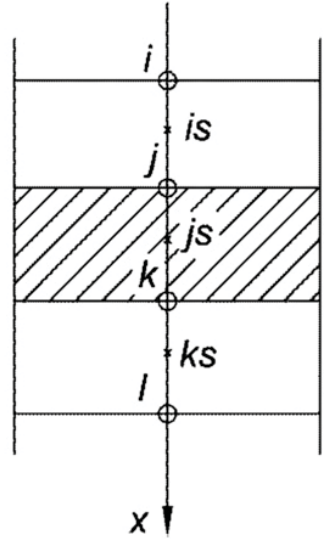

(b)
Fig. 3. (a) Typical control volume surrounding Point j; (b) control volume surrounding Point $j s$

solutions for two simple examples is shown in (Fig. 4). In the first example, a 10-m water head applied to a 100-mm-thick layer of soil with a hydraulic conductivity of $1 \times 10^{-2} \mathrm{~m} / \mathrm{s}$ was considered under steady-state flow conditions. The result shows that, although the nonlinear solution does not provide the exact solution, the nonlinear solution converges immediately, whereas the linear solution requires many iterations. With this simple example, the exact solution can be calculated using the approach proposed by Ergun (1952). The iteration loop is required for both linear and nonlinear solutions. The iteration accounts for determining the correct pressure field, which is initially unknown. The second example demonstrates the simulation of a system where the base soil is not erodible. Particle size distributions (PSDs) for both base soil and filter are shown in Fig. 4(b). Because the controlling constriction size of the filter is smaller than the representative base soil particles, there is no erosion of base soils. Therefore, the slurry does not contain any base particles, and hence no accumulation of base particles occurs within filter layers. The particle size distributions of a system are not changed, so the flow keeps stable during the simulation shown in Fig. 4(c). 

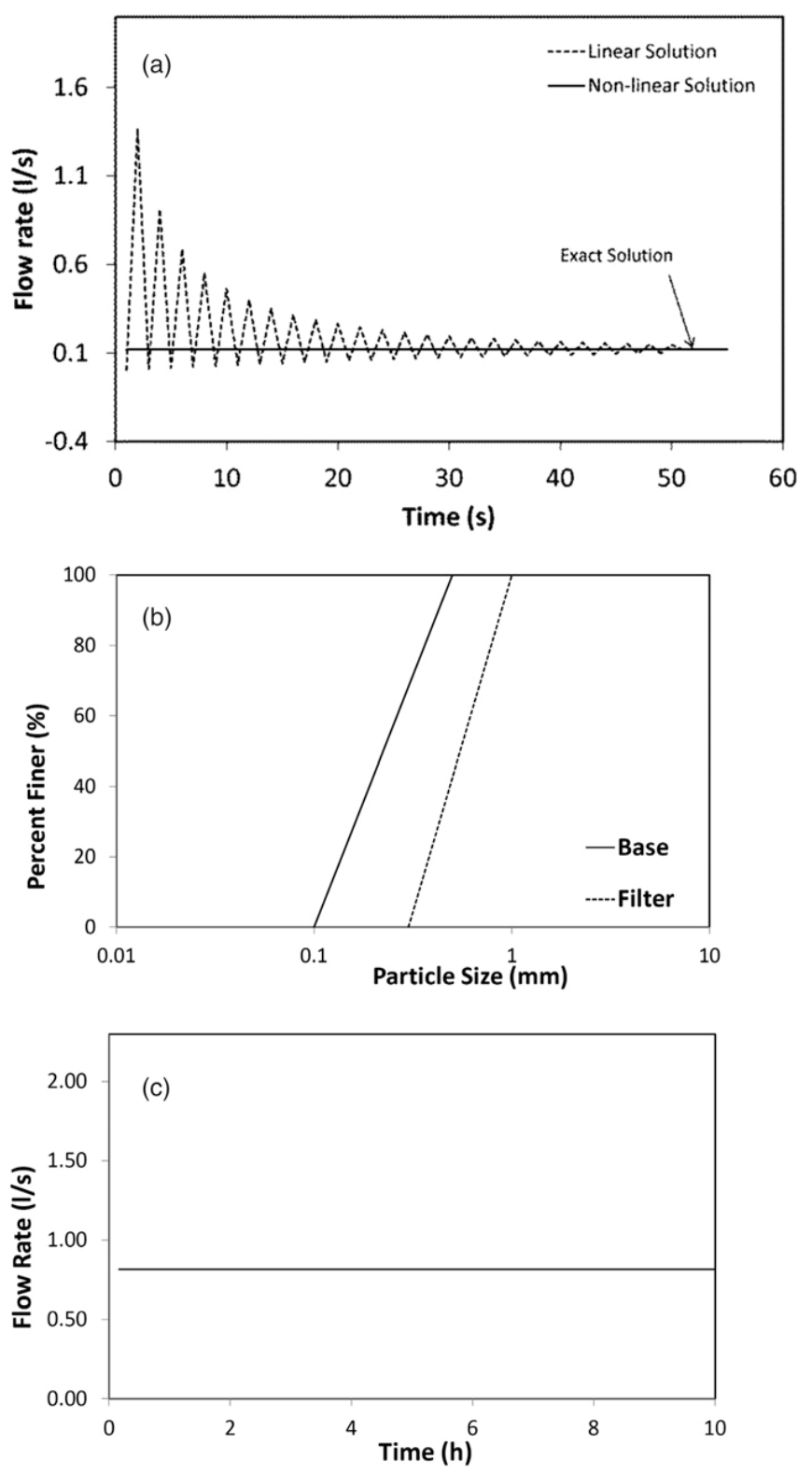

Fig. 4. (a) Comparison of linear and nonlinear solutions; (b) particle size distributions of initial base and filter soils; (c) flow rate where there is no erosion of base soil

Based on the pressure and velocity fields obtained from the preceding procedure, Eq. (12) can be solved, and the densities of mixture can be determined at any grid points within the filter medium at any time step. Accordingly, one can have an equation system written as

$$
\begin{aligned}
& -\left(g z_{i}+\frac{u_{i}^{2}}{2}\right) u_{i} \epsilon_{i} \rho_{i}+\left(g z_{i+1}+\frac{u_{i+1}^{2}}{2}\right) u_{i+1} \epsilon_{i+1} \rho_{i+1} \\
& =p_{i} \epsilon_{i} u_{i}-p_{i+1} \epsilon_{i+1} u_{i+1}-\frac{\mu_{s}}{\mu} u_{i s} \sum_{m=1}^{n_{p}} F_{m}
\end{aligned}
$$

Eq. (12) is mathematically derived based on the work-energy principle within an arbitrary control volume formed by two sections that are assigned indexes $i$ and $i+1$ [Fig. 1(b)]. It is true that
Eq. (23) is the same as Eq. (12), but it is presented in a numerical form applied to the discretized filter medium. The control volume of Eq. (12) is now Layer $i$ formed by two sections crossing Points $i$ and $i+1$ [Fig. 2(b)]. Therefore, Index $i$ in Eq. (12) denotes arbitrary Section $i$ [Fig. 1(b)], but $i$ in Eq. (23) denotes Point $i$. $u_{L i}$ denotes flow velocity within Layer $i$ [Fig. 1(b)], and $u_{i s}$ denotes flow velocity of Layer $i$ within filter media, i.e., the velocity at staggered point between Points $i$ and $i+1$. In brief, this numerical scheme includes the governing equations that represent the following salient aspects:

1. Flow condition (laminar and turbulent state): Navier-Stokes equation [Eq. (1)]; and

2. Accumulation and erosion of base soil via variation of slurry density and PSD, i.e., work energy balance concept [Eq. (23)] and constriction size concept by Raut and Indraratna (2008). All base particles whose sizes are smaller than the controlling constriction size of filters are eroded and transported into the filters. Therefore, this is the postulated criterion for initiating the erosion of base soils. In the corresponding analysis, Eq. (12) in the mathematical description [or Eq. (23) in the numerical phase] governs the erosion rates within the filter as represented by associated slurry densities. This equation considers the essential hydraulic conditions (pressures, flow velocities) and geometric conditions (constriction size of filter), thereby indirectly considering the effect of hydraulic gradient on the rate of erosion.

In the algorithm, the critical hydraulic gradient is represented by Eq. (23) that forms an integral component of the solution scheme (nonlinear). The erosion rate and hydraulic gradient is fundamental physics following the iterative forms of Darcy's law and NavierStokes equations that are directly captured in this algorithm.

\section{Comparison of Models with Experimental Data}

The aforementioned numerical procedures were integrated into a computational program using MATLAB software. The flowchart of the model programming is presented in Fig. 5. The proposed model was then compared with other existing filtration models presented by Indraratna and Vafai (1997), Locke et al. (2001), and Raut and Indraratna (2008). In brief, Indraratna and Vafai (1997) proposed a mathematical model that used the mass balance equation and Darcy's law to calculate the slurry density field within the media. The erodible base soil particles were governed by the structure of the pore network of filters. Indraratna and Vafai (1997) used a simple void model developed by Kovacs (1981), whereas Locke et al. (2001) adopted and modified a 3D pore model proposed earlier by Schuler (1996). The 3D pore model represents a network of spheres (pores) interconnected by pipes (pore constrictions). The input data for both Indraratna and Vafai (1997) and Locke et al. (2001) models include the following: (1) PSDs of the base soil and filter materials, (2) lengths of base soil and filter elements, and (3) hydraulic conditions such as the pressure head at the base soil-filter boundary. Indraratna and Vafai (1997) considered the effectiveness of a filter by assessing the change of PSDs within them, such that filters that can retain base soil particles may be judged as effective or vice versa. Locke et al. (2001) considered the effectiveness of filters based on the occurrence of the flow rate, whereas Raut and Indraratna (2008) suggested a constriction-based retention criterion to evaluate the filter using input data from the PSDs of base and filter soils. For convenience, the following acronyms are used to represent the current model, the Indraratna and Vafai (1997) model, the Locke et al. (2001) model, and the Raut and Indraratna (2008) model: CM, IVM, LM, and RIM, respectively. 


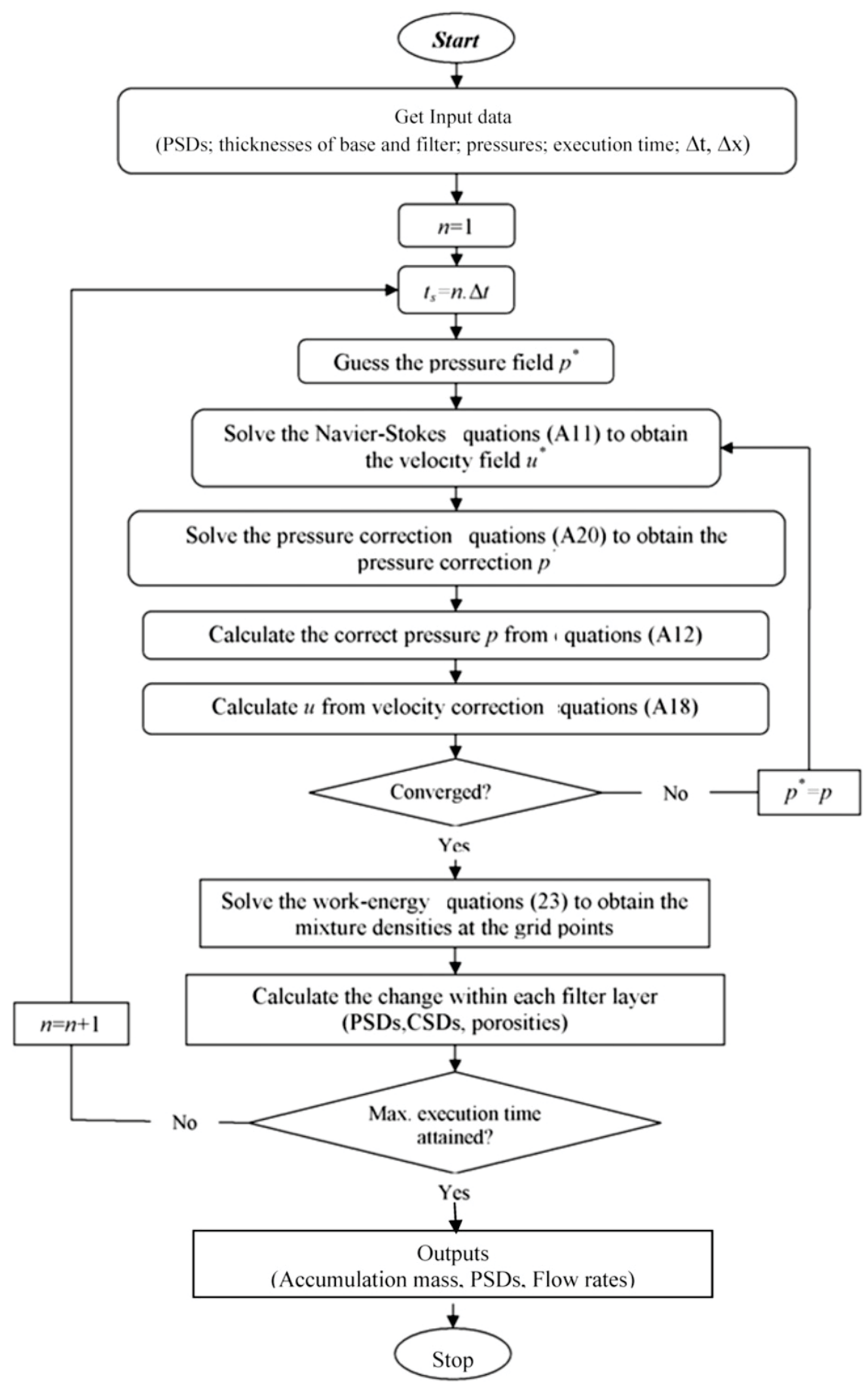

Fig. 5. Flowchart of programming

\section{Change of Particle Size Distributions of Filters in Various Depths}

Indraratna and Vafai (1997) and Locke et al. (2001) conducted comprehensive experiments for their model validation. The model of Locke et al. (2001) can consider the change in PSDs using an analytical approach, as well as measuring the PSD changes in the laboratory. These models were validated using the PSDs of filters after the test to determine the accumulation of base soil within filters at various depths. In each test containing two layers, the base soil layer was placed on top of the filter layer. The filter layer was divided into upper and lower layers. The tests conducted by Indraratna and Vafai (1997) considered uniform base and filter soils whose coefficients of uniformity were approximately 3 [Fig. 6(a)]. Further parameters of the tests including hydraulic conductivities of filter are shown in Table 1. The slurry minerals (suspended particles) include quartz, mica, and feldspar. After testing, the sample filter was divided into two 25 -mm-thick layers to analyze the PSDs. The retention of base soil within a filter can be expressed by the shift of the PSD within the filter. Obviously, the more the PSDs shifted to the left, the more the base soil accumulated within the filter. Because 

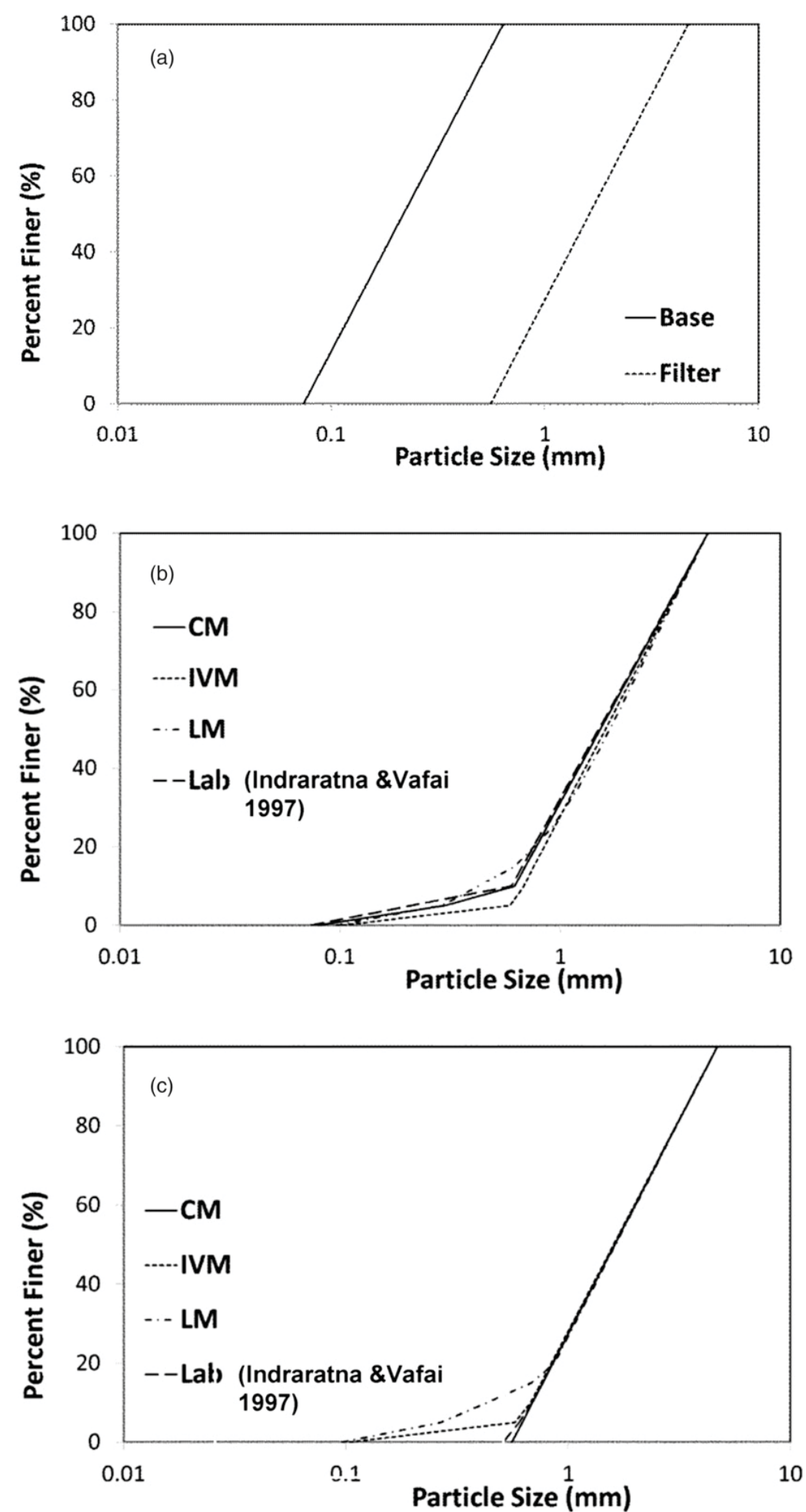

Fig. 6. (a) Particle size distributions of initial base and filter soils by Indraratna and Vafai (1997); filter particle size distributions after testing in (b) upper layer of filter and (c) lower layer of filter [CM = current model; IVM = Indraratna and Vafai (1997) model; LM = Locke et al. (2001) model; Laboratory $=$ laboratory observation]

the sizes of the particles of base soil accumulated within the filter were much smaller than those of the filter, the shift of the filter PSD to its lower region was more prominent than the original PSD. Figs. 6(b and c) show the PSDs of the upper and lower layers of the filter after the test, as well as the predictions from CM, IVM, and LM.
Similarly, Locke et al. (2001) considered the change of PSDs within filters after these tests. This testing program included two base-soil filter combinations where similar filters were used, but with different base soils [Fig. 7(a)]. The base soils used in this study were uniformly graded sand, whereas the filter was considered to be 
Table 1. Input Parameters of Filtration Tests

\begin{tabular}{lcccc}
\hline & $\begin{array}{c}\text { Indraratna and } \\
\text { Vafai (1997) }\end{array}$ & $\begin{array}{c}\text { Locke et al. } \\
(2001)\end{array}$ & $\begin{array}{c}\text { Raut and } \\
\text { Indraratna } \\
(2008)\end{array}$ & $\begin{array}{c}\text { Current } \\
\text { study }\end{array}$ \\
\hline $\begin{array}{l}\text { Input parameters } \\
\text { Thickness of }\end{array}$ & 100 & 150 & 50 & 50 \\
$\begin{array}{l}\text { Thickness } \\
\text { of filter (mm) }\end{array}$ & 100 & 800 & 100 & 100 \\
$\begin{array}{l}\text { Applied } \\
\text { hydraulic } \\
\text { head (kPa) }\end{array}$ & 9.8 & 14.7 & 250 & 250 \\
$\begin{array}{l}\text { Initial hydraulic } \\
\text { conductivities } \\
\text { (mm/s) }\end{array}$ & 2.5 & 139 & $0.09-163$ & $1.5-122$ \\
\hline
\end{tabular}

poorly graded. Parameters of the tests are shown in Table 1. After the tests, the filters were removed to obtain the PSDs at 50- and 200-mm depths from the interface between the base soil and the filter. Figs. 7(b-e) show the PSDs of filters after the tests, as well as the predictions from CM, IVM, and LM. As expected, the PSDs of the filter elements shifted from the left, which represented an accumulation of base soil particles within the filter.

IVM and LM used mass balance equations to calculate the slurry density within the filter. The mass balance in these models did not consider the effects of moving particles interacting or the pore network of the filters. The two models assumed erodible base soil particles that were so conservative in size compared with the laboratory observations. As pointed out by Indraratna et al. (2007), for a given filter soil, the size of erodible base soil particles can be represented by the controlling constriction size $\left(D_{c}\right)$ that can be
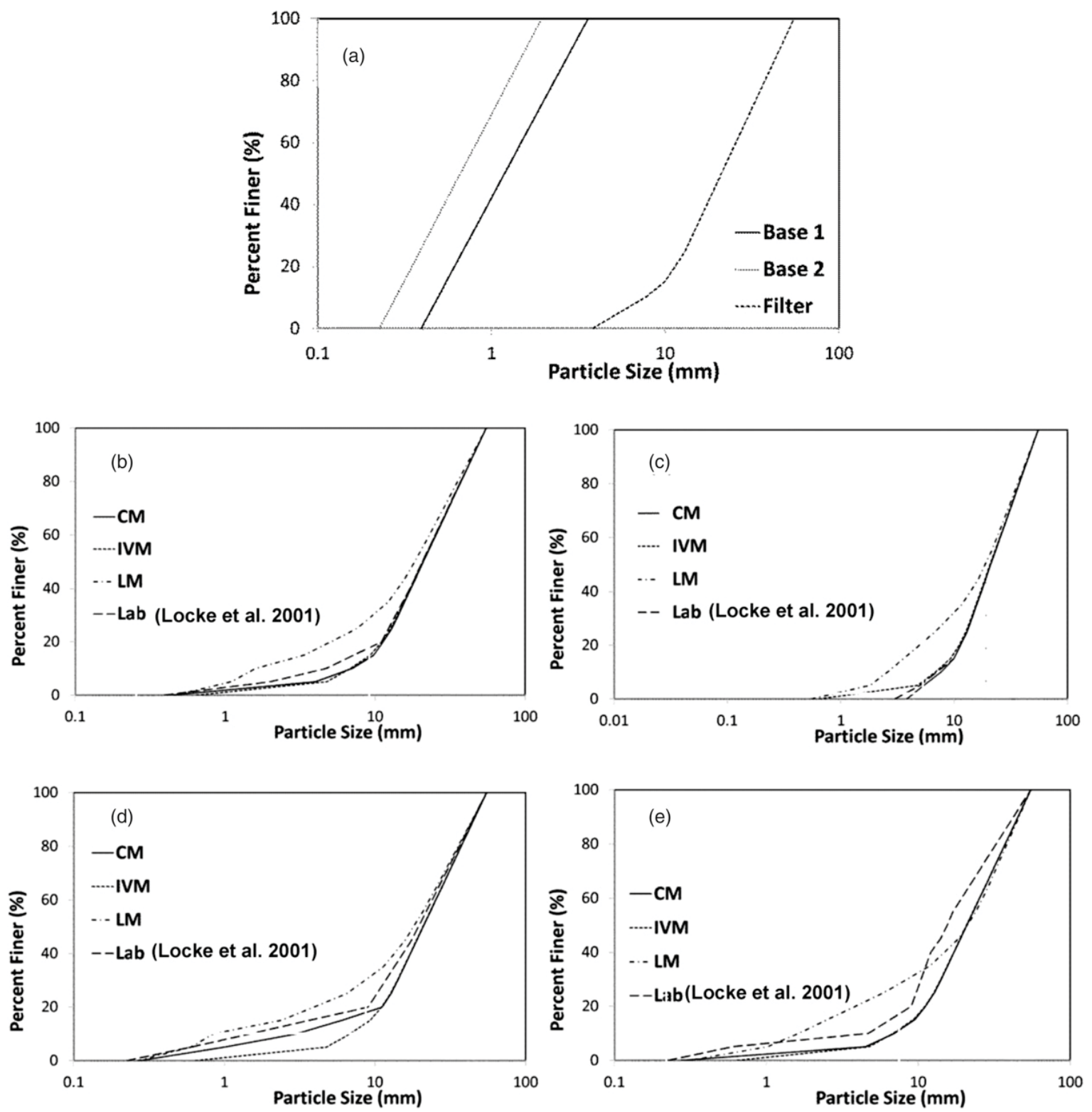

Fig. 7. (a) Particle size distributions of initial base and filter soils by Locke et al. (2001); particle size distributions of filter after testing (b) Base Soil 1-Filter at 50-mm depth, (c) Base Soil 1-Filter at 200-mm depth, (d) Base Soil 2-Filter at 50-mm depth, and (e) Base Soil 2-Filter at 200-mm depth $[C M=$ current model; IVM = Indraratna and Vafai (1997) model; LM = Locke et al. (2001) model; Laboratory = laboratory observation] 
determined using the filter PSD. The size of erodible base soil particles used in IVM (i.e., the equivalent diameter of pore channel $d_{0}$, calculated by Kovacs 1981) and in LM (i.e., the constriction size at which $95 \%$ were finer, $D_{c 95}$ ) is about two to three times the controlling constriction size. A conservative assumption can lead to a higher rate of erosion of base soil, as can the assumption that Darcy's law normally provides a higher velocity field than one caused by the turbulent effect (Cedergren 1989). When all these factors are combined, the distribution of the base soil and water mixture along the media can be overestimated, allowing particles of base soil to exist at any depth in the filters, even in effluent flow. For instance, Figs. 6 and 7 show the overpredicted accumulations of base soil particles that were not seen in the tests by IVM and LM at the lower layers for filters. Moreover, the accumulation of base soil particles within the filters predicted by LM were higher than the others because this model assumed that once base soil particles are retained, they cannot move any more.

\section{Flow Rates during Filtration}

The results of the tests conducted by Indraratna and Vafai (1997) and Locke et al. (2001) showed the change of filter PSDs at various depths through which one can determine the accumulation of base soil within the filters. However, this information may not be enough to evaluate the filtration mechanism that can be expressed by the flow rate during this process. Therefore, in this study, additional tests were conducted to obtain the flow rate during filtration. The test procedures were discussed elsewhere by Locke et al. (2001). It is noted that uncontaminated clear water was used initially for the filtration test. The slurry was formed after the base soil particles eroded and then were mixed with water. The slurry densities can be obtained using Eq. (12) in the mathematical model [or Eq. (23) in the numerical phase[. Once the densities are known, the other relevant parameters (volumetric concentration and water content) can then be determined. All the tests used the same base soil (B). The first series was conducted using uniform filters (F1, F2, and F3). The second series used moderately graded filters (F4, F5, and F6), and the last series was conducted with a poorly graded filter (F7; Fig. 8). Further parameters of the tests can be referred to in Table 1 . The results then were compared with the predictions from CM, IVM, and LM (Fig. 9). The observations from the tests showed that combinations of B-F1, B-F2, and B-F4 provide effective filters where the flow rate slowed initially and then became steady, a tendency that can be attributed to the formation of a stable internal self-filtering layer. Meanwhile, the combinations of B-F3, B-F5, and B-F6 proved to be ineffective filters because the pore space of the filters was large compared with the particles of base soil; hence, the base soils were eroded into the filter, causing an initial reduction in the flow rate. Subsequently, an unstable formation of self-filtering layers was temporarily formed where the base soil particles in these layers were then washed out, leading to an increase in the flow rate at a later stage, and afterward, the flow rate stabilized. The combination of B-F7 showed a significant reduction in the flow rate, which was attributed to the potential for clogging within the filter. The IVM and LM models seemed to have limitations in describing the timedependent flow rate because the trend of the flow rates observed by these models were different from the laboratory observations. As mentioned earlier, all the simulations of IVM and LM showed that there was a washout of base soil. Therefore, the flow rate predictions using IVM and LM were similar. At the first stage, the flow rate increased because of erosion of base soil particles, but then it stabilized as erosion ceased.

The change in PSD cannot be solely used to evaluate filtration effectiveness. For a residual base soil tested by Indraratna et al. (1996), it was possible to evaluate clogging by observing a continually downward trend in the laboratory flow rate that could not attain an apparent steady state even after many hours of testing. However, for most base soils, such clogging often takes a long time to occur, and the same test may need to be run for several months without interruption. It may be proposed that by detailed examination of the fines trapped in the filter PSD after a substantial period of testing, the risk of clogging may be quantified, but this has been beyond the scope of this particular study. Therefore, the empirical evaluation of clogging based on the change of is not discussed here.

\section{Comparison with Constriction-Based Retention Criteria}

Raut and Indraratna (2008) proposed a constriction-based retention criterion $\left(D_{35}^{c} / d_{85}^{*}=1\right)$ (where $D_{35}^{c}=$ controlling constriction size of the filter, and $d_{85}^{*}=$ particle size taken from the degraded base soil PSD at which $85 \%$ of the particles are finer, after the filtration test) to assess the effectiveness of filters. In this criteria, if $D_{35}^{c} / d_{85}^{*}>1$, the filter is considered to be ineffective. A total of 14 filtration test data were adopted in this section for comparison (seven filtration tests from Raut and Indraratna 2008 and seven tests conducted in this study as shown in Table 2). Raut and Indraratna's criteria are

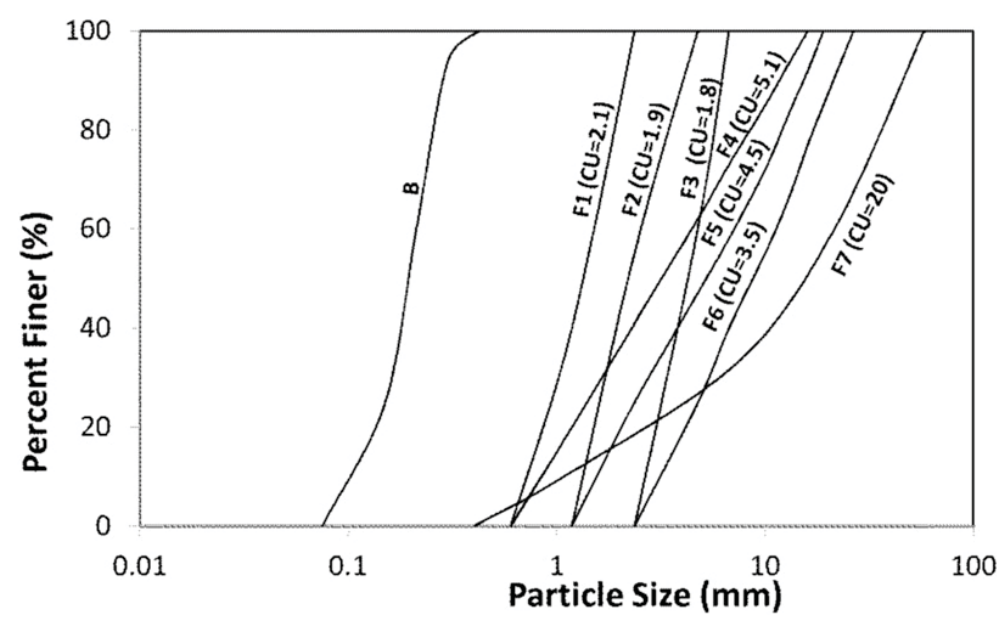

Fig. 8. Particle size distributions of base soil and filters used in current study 

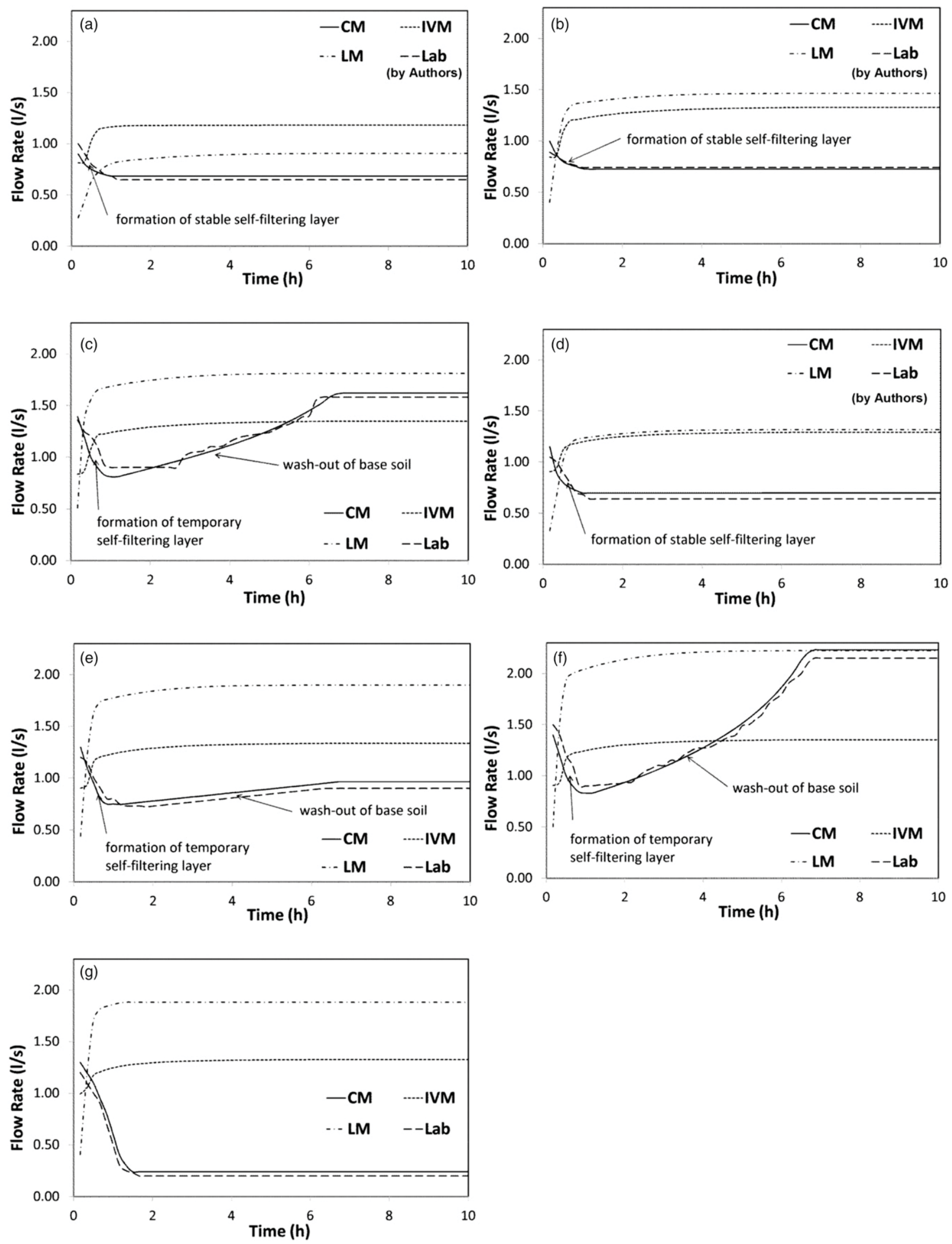

Fig. 9. Flow rate of Combinations (a) B-F1, (b) B-F2, (c) B-F3, (d) B-F4, (e) B-F5, (f) B-F6, and (g) B-F7 [CM = current model; IVM = Indraratna and Vafai (1997) model; LM = Locke et al. (2001) model; Laboratory = laboratory observation conducted by the authors] 
Table 2. Comparison with Raut and Indraratna (2008) model

\begin{tabular}{lllll}
\hline ID & $D_{35}^{c} / d_{85}^{*}($ RIM) & \multicolumn{1}{c}{ CM } & $\begin{array}{c}\text { Laboratory. } \\
\text { assessment }\end{array}$ & \multicolumn{1}{c}{ Reference } \\
\hline 1 & 0.63 (Stable) & Stable & Stable & Raut and Indraratna (2008) \\
2 & 1.13 (Unstable) & Stable & Stable & \\
3 & 2.29 (Unstable) & Unstable & Unstable & \\
4 & 0.83 (Stable) & Stable & Stable & \\
5 & 1.01 (Unstable) & Stable & Stable & \\
6 & 1.17 (Unstable) & Stable & Stable & \\
7 & 1.14 (Unstable) & Stable & Unstable & \\
8 & 0.66 (Stable) & Stable & Stable & Current study \\
9 & 1.17 (Unstable) & Stable & Stable & \\
10 & 2.28 (Unstable) & Unstable & Unstable & \\
11 & 0.74 (Stable) & Stable & Stable & \\
12 & 1.38 (Unstable) & Unstable & Unstable & \\
13 & 2.83 (Unstable) & Unstable & Unstable & \\
14 & 1.11 (Unstable) & Stable & Stable & \\
\hline
\end{tabular}

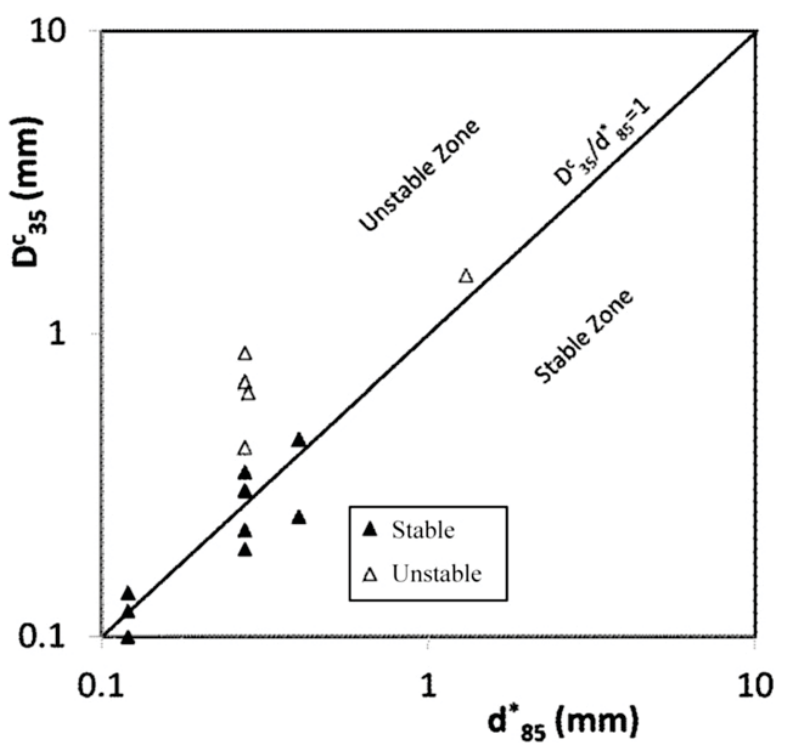

Fig. 10. Predictions using Raut and Indraratna (2008) model and the authors' laboratory observations

applicable in most cases. Four cases predicted by RIM do not agree with the laboratory observations. This shows that the model provided in this study has better accuracy. Whereas the filters for Cases 2, 5, 6, and 9 are considered to be ineffective using RIM, the assessments from the laboratory observations state that these filters are effective (Fig. 10). The discrepancy between the observation and RIM occurred when the ratios of $D_{35}^{c} / d_{85}^{*}$ were close to the boundary (i.e., slightly greater than 1). This statement showed that the boundary for the ratio $D_{35}^{c} / d_{85}^{*}$ for assessing the effectiveness of the filter was slightly conservative.

\section{Conclusions}

Base soil particles undergoing seepage flow can be eroded and transported into the filter environment. Given the nature of pore spaces in the filter and the internal hydrodynamic conditions, these particles may be retained (filtered) or washed out of the filter, making it either effective or ineffective. The filtration process is a time-dependent process affected by factors including the size of the base soil particles, constriction sizes of the filters, and hydrodynamic conditions. This study proposed an analytical model to capture these factors. It used the Navier-Stokes equations for porous media to capture the hydrodynamic flow via nonlinear equations. The revised numerical procedure to solve the Navier-Stokes equations in nonlinear form in this study showed features that were superior to the linear form. The work-energy equation incorporating the effect of the controlling constriction size could further explain the phenomena of accumulation and redistribution of base soil in the filter.

A series of laboratory tests conducted in this study and data sourced from previous studies were used to verify the model. By considering the changes in particle size distributions at various depths within the filters, and the flow rates during the filtration process, the current model could offer reasonable predictions that compared well with the experimental data. It was found that two filtration models proposed by Indraratna and Vafai (1997) and Locke et al. (2001) overpredict the PSDs of filters at various layers because of simplified assumptions. These models cannot capture the condition of water flow that changes because of the forming of stable self-filtering layers or temporary self-filtering layers within filter media. In contrast, the current model provides better predictions in terms of the accumulation of base soils within filters and the flow conditions during the filtration process. The proposed model also captures the prediction of the system hydraulic conductivity after a stable filter zone is established. In addition, the assessment of filter effectiveness based on the proposed model provides similar results to the criteria of Raut and Indraratna (2008), but the latter cannot capture the time-dependent filtration process.

\section{Appendix. Revised Algorithm for the Navier-Stokes Equation}

The nonlinear form of discretization equations of Eq. (22) can be given as follows:

$$
\begin{aligned}
& a_{j j} u_{j s}^{2}+a_{j k} u_{j s} u_{k s}+a_{j i} u_{j s} u_{i s}+a_{j} u_{j s}+a_{k} u_{k s}+a_{i} u_{i s}+b_{j} \\
& \quad+\left[p_{j}-p_{k}\right] B_{j}=0
\end{aligned}
$$

where

$$
\begin{gathered}
a_{j j}=\rho_{w} \Delta t \epsilon_{j s} \frac{l_{k}}{l_{j}+l_{k}}-\rho_{w} \Delta t \epsilon_{j s} \frac{l_{i}}{l_{i}+l_{j}}+\Delta t l_{j} \frac{1-\epsilon_{j s}}{d^{2} \epsilon_{j s}^{2}} 1.75 \rho_{w} \bar{d} \\
a_{j k}=\rho_{w} \Delta t \epsilon_{j s} \frac{l_{j}}{l_{j}+l_{k}} \\
a_{j i}=-\rho_{w} \Delta t \epsilon_{j s} \frac{l_{j}}{l_{i}+l_{j}} \\
a_{j}=\rho_{w} l_{j} \epsilon_{j s}+2 \mu \epsilon_{j s} \Delta t \frac{1}{l_{j}+l_{k}}+2 \mu \epsilon_{j s} \Delta t \frac{1}{l_{i}+l_{j}} \\
+\Delta t l_{j} \frac{1-\epsilon_{j s}}{d^{2} \in \epsilon_{j s}^{2}} 150\left(1-\epsilon_{j s}\right) \mu \\
a_{k}=-2 \mu \epsilon_{j s} \Delta t \frac{1}{l_{j}+l_{k}}
\end{gathered}
$$




$$
\begin{gathered}
a_{i}=-2 \mu \epsilon_{j s} \Delta t \frac{1}{l_{i}+l_{j}} \\
b_{j}=-\rho_{w} l_{j} \epsilon_{j s} u_{j s}^{0} \\
B_{j}=-\epsilon_{j s} \Delta t
\end{gathered}
$$

In these expressions $u, p, \in$, and $l=$ velocity, pressure, porosity, and length of layer, respectively (subscripts denote the indexes for locations); $g=$ acceleration from gravity; $\rho_{w}=$ density of water; and $d=$ diameter of the particles occurring in the element. For the boundary points (i.e. Point $1 s$ and Point $n s$ ), the discretization equations can be obtained by assuming that the velocities at Points 1 and $1 s$ are the same. Similarly, the velocities at Points $n s$ and $n+1$ are the same.

The nonlinear equation systems [Eq. (24)] can only be solved when the pressure field is given or estimated. Once a pressure field is given, the solution for nonlinear equation systems can be solved using Newton's method (Grosan and Abraham 2008) by treating equations as a nonlinear equation system

$$
f_{i}\left(u_{1 s}, u_{2 s}, \ldots, u_{n s}\right)=0 \quad(i=1 / n)
$$

A guessed pressure field $p^{*}$ applied to the equation system [Eq. (24)] leads to a velocity field denoted by $u^{*}$, which can be obtained by solving the following equations:

$$
\begin{aligned}
& a_{j j}\left(u_{j s}^{*}\right)^{2}+a_{j k} u_{j s}^{*} u_{k s}^{*}+a_{j i} u_{j s}^{*} u_{i s}^{*}+a_{j} u_{j s}^{*}+a_{k} u_{k s}^{*} \\
& \quad+a_{i} u_{i s}^{*}+b_{j}+\left[p_{j}^{*}-p_{k}^{*}\right] B_{j}=0
\end{aligned}
$$

However, unless the correct pressure field is used, the resulting velocity field cannot satisfy the continuity equation [Eq. (4)]. Therefore, to obtain a correct velocity field, a guessed pressure field should be chosen so that the velocity field is closer to the satisfied continuity equation. The revised algorithm in this study is similar to the equation proposed by Patankar (1980)

$$
p=p^{*}+p^{\prime}
$$

where $p^{\prime}=$ pressure correction. Subsequently, the change in pressure [Eq. (35)] results in a change of velocity

$$
u=u^{*}+u^{\prime}
$$

where $u^{\prime}=$ velocity correction.

Substitution of Eqs. (35) and (36) into Eq. (24) leads to

$$
\begin{aligned}
& a_{j j}\left(u_{j s}^{*}+u_{j s}^{\prime}\right)^{2}+a_{j k}\left(u_{j s}^{*}+u_{j s}^{\prime}\right)\left(u_{k s}^{*}+u_{k s}^{\prime}\right) \\
& \quad+a_{j i}\left(u_{j s}^{*}+u_{j s}^{\prime}\right)\left(u_{i s}^{*}+u_{i s}^{\prime}\right)+a_{j}\left(u_{j s}^{*}+u_{j s}^{\prime}\right) \\
& \quad+a_{k}\left(u_{k s}^{*}+u_{k s}^{\prime}\right)+a_{i}\left(u_{i s}^{*}+u_{i s}^{\prime}\right)+b_{j} \\
& \quad+\left[\left(p_{j}^{*}+p_{j}^{\prime}\right)-\left(p_{k}^{*}-p_{k}^{\prime}\right)\right] B_{j}=0
\end{aligned}
$$

To determine the pressure correction $p^{\prime}$, two equation systems [Eqs. (34) and (37)] must be solved by incorporating the mass balance equation [Eq. (4)]. At this point, subtracting Eq. (37) from Eq. (34) and dropping the higher-order terms

$$
a_{j} u_{j s}^{\prime}+a_{k} u_{k s}^{\prime}+a_{i} u_{i s}^{\prime}+\left[p_{j}^{\prime}-p_{k}^{\prime}\right] B_{j}=0
$$

The assumption $u_{j s}^{\prime}=u_{k s}^{\prime}=u_{i s}^{\prime}$ makes Eq. (38) simpler as follows:

$$
u_{j s}^{\prime}=D_{j}\left[p_{j}^{\prime}-p_{k}^{\prime}\right]
$$

where

$$
D_{j}=-B_{j} /\left(a_{j}+a_{k}+a_{i}\right)
$$

Eqs. (36) and (39) can be rewritten as

$$
u_{j s}=u_{j s}^{*}+D_{j}\left[p_{j}^{\prime}-p_{k}^{\prime}\right]
$$

The continuity equation [Eq. (4)] can be solved to obtain discretization equations based on the control volume chosen in Fig. 3(a). Thus

$$
\epsilon_{j s} u_{j s}=\epsilon_{i s} u_{i s}
$$

If all the velocity components can be substituted [Eq. (39)], the following discretization equations for the pressure corrections can be obtained:

$$
a_{I} p_{i}^{\prime}+a_{J} p_{j}^{\prime}+a_{K} p_{k}^{\prime}=b_{J}
$$

where

$$
\begin{gathered}
a_{I}=-\epsilon_{i s} D_{i} \\
a_{K}=-\epsilon_{j s} D_{j} \\
a_{J}=\epsilon_{i s} D_{i}+\epsilon_{j s} D_{j} \\
b_{J}=\epsilon_{i s} u_{i s}^{*}-\epsilon_{j s} u_{j s}^{*}
\end{gathered}
$$

The boundary for the pressure-correction equations [Eq. (43)] provides pressure at the boundary, which means that a guessed pressure field $\left(p^{*}\right)$ can be determined such that, at a boundary, the guessed value of pressure has known values. In other words, $p^{\prime}$ at a boundary will be zero. The pressure correction suggested above [Eq. (43)] offers a converged solution for the nonlinear equation system [Eq. (24)]. However, to improve the speed of convergence, Eq. (35) can be modified using a relaxation factor $\alpha$

$$
p=p^{*}+\alpha p^{\prime}
$$

A relaxation factor $\alpha=10$ was found to be satisfactory in a large number of trials and was adopted in this study.

\section{Notation}

The following symbols are used in this paper:

$C_{d}=$ drag coefficient;

$C_{u}=$ coefficient of uniformity of soil;

$C_{v}=$ slurry volumetric concentration;

$D_{35}^{c}=$ controlling constriction size of the filter;

$d=$ diameter of the particles existing within the element;

$d_{85}^{*}=$ particle size taken from the degraded base soil PSD at which $85 \%$ of the particles are finer;

$E=$ energy;

$e=$ void ratio of soil;

$F_{m}=$ hydrodynamic force on the $m$ th particle within a discretized layer;

$f_{b}=$ body force per unit volume; 
$g=$ acceleration due to gravity;

$k=$ saturated hydraulic conductivity of soils;

$l_{i}=$ length corresponding to a discretized layer;

$m=$ number of unit layers of a flow path;

$n=$ porosity of soil;

$n_{b}=$ number of base soil layers in a discretized medium;

$n_{f}=$ number of filter soil layers in a discretized medium;

$P_{j}=$ percentage of particle having diameter $d_{j}$ within slurry;

$p=$ pressure of water;

$p^{*}=$ guessed pressure;

$p^{\prime}=$ pressure correction;

$S_{p}=$ projected area;

$u=$ velocity of water flow;

$u^{*}=$ velocity determined using guessed pressure $p^{*}$;

$u^{\prime}=$ velocity correction;

$u_{i}=$ velocity of water at Point $i$ or Section $i$;

$u_{L i}=$ velocity of water within a discretized layer;

$V_{i n}^{s}=$ incoming volumes of slurry of a discretized layer;

$V_{i n}^{w}=$ incoming volumes of water of a discretized layer;

$W=$ work done;

$W_{i n}^{p}=$ incoming mass of soil particles of a discretized layer;

$W_{i n}^{s}=$ incoming mass of slurry of a discretized layer;

$W_{i n}^{w}=$ incoming mass of water of a discretized layer;

$W_{j}^{p}=$ mass of soil corresponding to a specific diameter $d_{j}$ accumulated within a discretised layer;

$W_{\text {lmax }}=$ maximum weight of particles of the fine part retained in the voids between the coarse part;

$W_{o u t}^{p}=$ outgoing mass of soil particles of a discretized layer;

$W_{\text {out }}^{s}=$ outgoing mass of slurry of a discretized layer;

$W_{p}=$ pressure work;

$W^{p}=$ total mass of soil particles remaining in a discretized layer;

$W_{s}=$ shear work;

$x=$ distance;

$\beta=$ coefficient used to determine $f_{b}$;

$\Delta t=$ time interval;

$\epsilon=$ porosity of soil used in the Navier-Stokes equations for porous media;

$\mu=$ viscosity of water;

$\mu_{s}=$ viscosity of slurry;

$\rho=$ density of slurry;

$\rho_{i}=$ density of slurry at Point $i$ or Section $i$; and

$\rho_{w}=$ density of water.

\section{References}

Bouillard, J. X., Lyczkowski, R. W., and Gidaspow, D. (1989). "Porosity distributions in a fluidized bed with an immersed obstacle." AIChE J., 35(6), 908-922.

Brenner, H. (1958). "Dissipation of energy due to solid particles suspended in a viscous liquid." Phys. Fluids, 1(4), 338-346.

Cedergren, H. R. (1989). Seepage, drainage, and flow nets, 3rd Ed., Wiley, New York.

Ergun, S. (1952). "Fluid flow through packed columns." Chem. Eng. Prog., 48(2), 89-94.
Fannin, J. (2008). "Karl Terzaghi: From theory to practice in geotechnical filter design." J. Geotech. Geoenviron. Eng., 134(3), 267-276.

Grosan, C., and Abraham, A. (2008). "A new approach for solving nonlinear equations systems." IEEE Trans. Syst. Man Cybern. A Syst. Hum., 38(3), 698-714.

Happel, J., and Brenner, H. (1965). Low Reynolds number hydrodynamics, with special applications to particulate media, Prentice Hall, New York.

Holland, F. A., and Bragg, R. (1995). Fluid flow for chemical engineers, 2nd Ed., Edward Arnold, London.

Honjo, Y., and Veneziano, D. (1989). "Improved filter criterion for cohesionless soils." J. Geotech. Eng., 115(1), 75-94.

Humes, C. (1996). "A new approach to compute the void-size distribution curves of protective filters." Geofilters'96, J. Lafleur and A. L. Rollin, eds., Bitech, Montreal.

Indraratna, B., Raut, A. K., and Khabbaz, H. (2007). "Constriction-based retention criterion for granular filter design.” J. Geotech. Geoenviron. Eng., 133(3), 266-276.

Indraratna, B., and Vafai, F. (1997). "Analytical model for particle migration within base soil-filter system." J. Geotech. Geoenviron. Eng., 123(2), 100-109.

Indraratna, B., Vafai, F., and Dilema, E. L. G. (1996). "An experimental study of the filtration of a lateritic clay slurry by sand filters." Geotech. Eng., 119(2), 75-83.

Kenney, T. C., Chahal, R., Chiu, E., Ofoegbu, I., Omange, G. N., and Ume, C. A. (1985). "Controlling constriction sizes of granular filters." Can. Geotech. J., 22(1), 32-43.

Kovacs, G. (1981). Seepage hydraulics, Elsevier Scientific, Amsterdam, Netherlands.

Lafleur, J., Mlynarek, J., and Rollin, A. L. (1989). "Filtration of broadly graded cohesionless soils.” J. Geotech. Eng., 115(12), 1747-1768.

Locke, M., Indraratna, B., and Adikari, G. (2001). "Time-dependent particle transport through granular filters." J. Geotech. Geoenviron. Eng., 127(6), 521-529.

Patankar, S. V. (1980). Numerical heat transfer and fluid flow, Hemisphere, Washington, DC.

Raut, A. K., and Indraratna, B. (2008). "Further advancement in filtration criteria through constriction-based techniques." J. Geotech. Geoenviron. Eng., 134(6), 883-887.

Schuler, U. (1996). "Scattering of the composition of soils-An aspect for the stability of granular filters." Geofilters'96, J. Lafleur and A. L. Rollin, eds., Bitech, Montreal.

Sherard, J. L., and Dunnigan, L. P. (1989). "Critical filters for impervious soils." J. Geotech. Eng., 115(7), 927-947.

Sherard, J. L., Dunnigan, L. P., and Talbot, J. R. (1984a). "Basic properties of sand and gravel filters." J. Geotech. Eng., 110(6), 684-700.

Sherard, J. L., Dunnigan, L. P., and Talbot, J. R. (1984b). "Filters for silts and clays." J. Geotech. Eng., 110(6), 701-718.

Silveira, A., de Lorena Peixoto, T., and Nogueira, J. (1975). "On void size distribution of granular materials." Proc., 5th Pan-American Conf. on Soil Mechanics and Foundation Engineering, Sociedad Argentina de Ingeniería Geotécnica, Buenos Aires, Argentina, 161-176.

Street, R. L., Watters, G. Z., and Vennard, J. K. (1996). Elementary fluid mechanics, 7th Ed., Wiley, New York.

Tannehill, J. C., Anderson, D. A., and Pletcher, R. H. (1997). Computational fluid mechanics and heat transfer, 2nd Ed., Taylor \& Francis, Philadelphia.

Tsuji, Y., Kawaguchi, T., and Tanaka, T. (1993). "Discrete particle simulation of two-dimensional fluidized bed." Powder Technol., 77(1), 79-87.

Vaughan, P. R., and Soares, H. F. (1982). "Design of filters for clay cores of dams." J. Geotech. Eng. Div., 108(GT1), 17-31.

Witt, K. J. (1993). "Reliability study of granular filters." Filters in geotechnical and hydraulic engineering, J. Brauns, M. Heibaum, and U. Schuler, eds., Balkema, Rotterdam, Netherlands.

Wittmann, L. (1979). "The process of soil-filtration, its physics and the approach in engineering practice." Proc., 7th European Conf. on Soil Mechanics and Foundation Engineering, British Geotechnical Association, London. 